FERMILAB-TM-1468

\title{
Resistor Cooling in a Vacuum
}

\author{
R. Crittenden and J. Krider \\ Research Facilities Department \\ Fermi National Accelerator Laboratory \\ P.O. Box 500, Batavia, Illinois 60510
}

July 31,1987 


\section{Resistor Cooling in a Vacuum \\ R. Crittenden and J. Krider \\ Research Facilities Department}

This note describes thermal measurements which were done on a resistor operating both in air at one atmosphere pressure and in a vacuum of a few millitorr. The motivation for this measurement was our interest in operating a BGO crystal-photomultiplier tube-base assembly in a vacuum, as a synchrotron radiation detector to tag electrons in the MT beam. We wished to determine what fraction of the total resistor power was dissipated by convection in air, in order to know whether there would be excessive heating of the detector assembly in a vacuum.

A $2.9 \Omega$ 1/2 watt carbon resistor was connected by one inch long leads to a BNC connector. A type $T$ thermocouple was attached with epoxy to the surface of the resistor to monitor its temperature. The assembly was placed in an evacuable chamber. A 1.20 Volt power supply operated the resistor at $1 / 2$ watt total power dissipation.

The first measurement was done at atmospheric pressure. Figure 1 shows the output from a chart recorder which was monitoring the thermocouple voltage. The horizontal scale is $6 \mathrm{sec} / \mathrm{smallest}$ division, and the vertical scale is nearly linear at $1 \mathrm{c} /$ smallest division. The heating time constant is $35 \mathrm{sec}$, and the maximum temperature rise is $15 \mathrm{C}^{\circ}$.

The second measurement was done at a pressure of 3 milliTorr. Figure 2 shows the chart recorder output for this measurement. The scales are the same as in Figure 1. The heating time constant is 75 $\mathrm{sec}$, and the maximum temperature rise is $31 \mathrm{C}^{\circ}$.

These measurements indicate that approximately half of the resistor cooling was by convection, which requires the presence of air. The other half was by radiation and conduction through the leads, which occurs equally well in a vacuum. This ratio between convection and the other forms of heat dissipation was measured only at the full rated power of the resistor. It was not determined experimentally whether the ratio is dependent on the amount of power being dissipated; however, the measurement does set an upper limit on the temperature rise of the resistor. Our conclusion is that the detector will operate in a vacuum at not more than twice the temperature rise that has been observed in air. In addition, potting of the resistor divider in the base should improve conduction cooling. 
We would like to thank Bob oudt and Mike Starr for providing the vacuum apparatus used in these measurements and Chuck Mangene for providing the thermocouple and chart recorder.

A simple linear model can be used to fit these data. We assume that the input power, $P$, is constant. The combined power loss to radiation and conduction, $P_{r c}$, is a function of the temperature difference, $\Delta T$, between the resistor and its surroundings. If power is lost only through radiation and conduction, then to first order $\Delta T_{1}=P_{r c}\left(\partial T / \partial P_{r c}\right)$. Let this thermal resistance $\partial T / \partial P_{r c}=R_{1}$.

Similarly, the power loss through convection to the air is a function of the temperature difference: $\Delta T_{2}=P_{v}\left(\partial T / \partial P_{v}\right)$. Let this thermal resistance $\partial T / \partial P_{V}=R_{2}$.

The incremental thermal energy, $\Delta q$, stored in the resistor when it is at a different temperature from its surroundings, is also a linear function of the temperature difference: $q=\Delta T(\partial q / \partial T)$. Let the specific heat $\partial q / \partial T=C$.

Using these definitions we draw by analogy the electronic circuit shown in Figure 3 to represent heat loss. In a vacuum $R_{2} \gg R_{1}$, so $R_{1}=\Delta T_{1} / P$. In air the net thermal resistance is $R_{12}=\Delta T_{12} / P$, where $R_{12}=R_{1} R_{2} /\left(R_{1}+R_{2}\right)$ allows $R_{2}$ to be calculated. The equilibrium temperature in air is $\Delta T_{12}=R_{12} P$.

The time constant in a vacuum is $\tau_{1}=R_{1} C$, so $C=\tau_{1} /\left(\Delta T_{1} / P\right)$. The time constant in air is $\tau_{12}=R_{12} C$. Using the measured value of $\tau_{1}$, the model predicts $\tau_{12}$ to be $36 \mathrm{sec}$, which is consistent with the measured value of $35 \mathrm{sec}$.

Using the measured values we find the following:

$R_{1}=62 \mathrm{C} /$ watt (radiation and conduction)

$\mathrm{R}_{2}=58 \mathrm{C}$ / watt (convection)

$\mathrm{R}_{12}=30 \mathrm{C} /$ watt (radiation, conduction and convection)

$\mathrm{C}=1.2$ joule $/ \mathrm{C}^{\circ}$ 


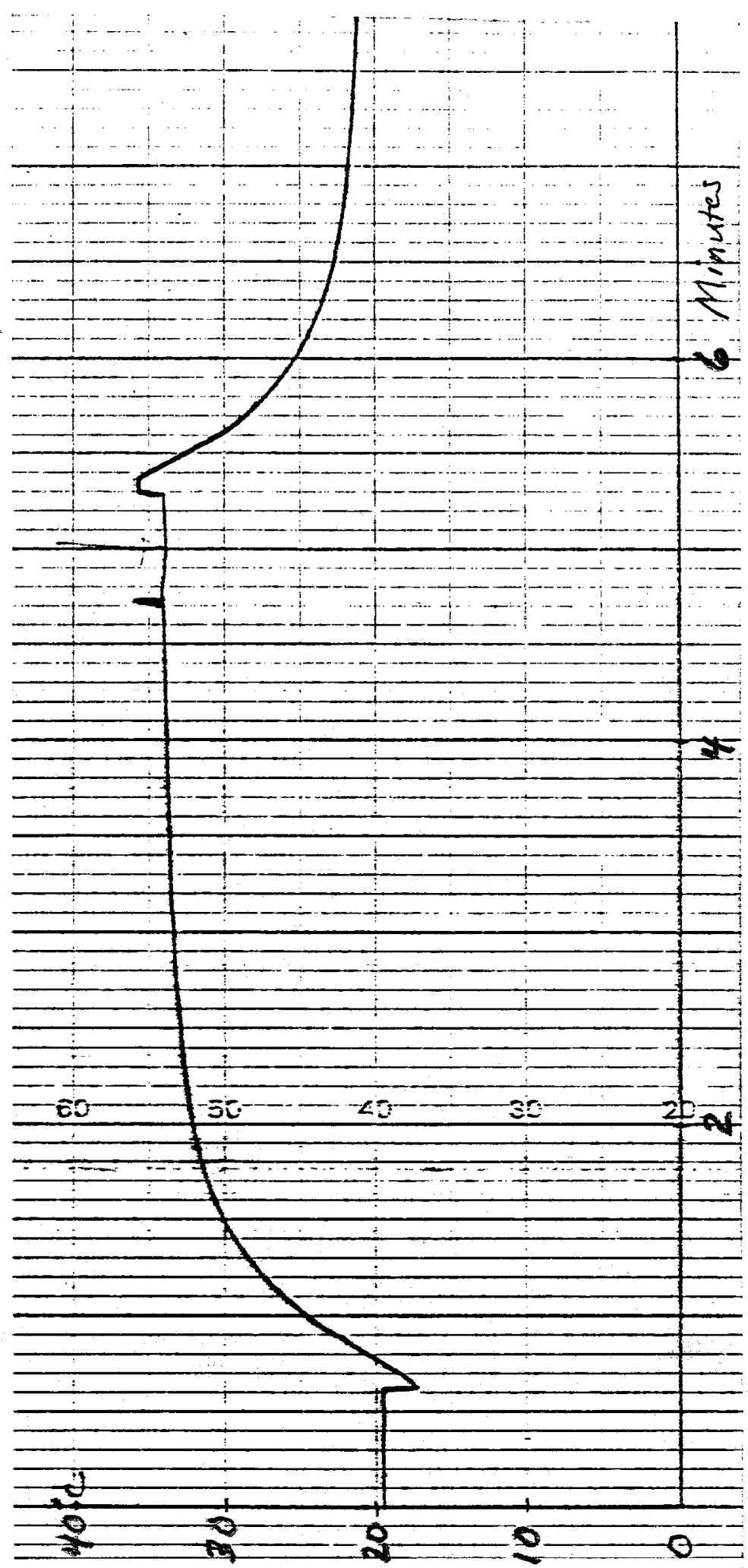




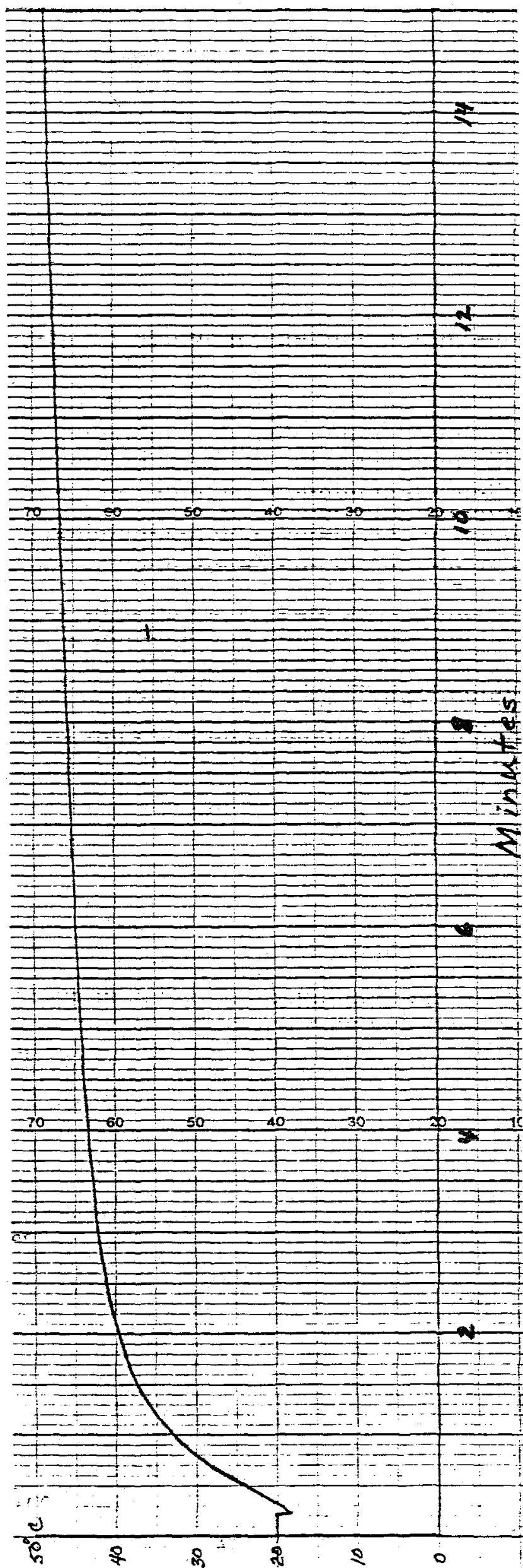


Figure 3 Electrical Analogy to Thermal Heating

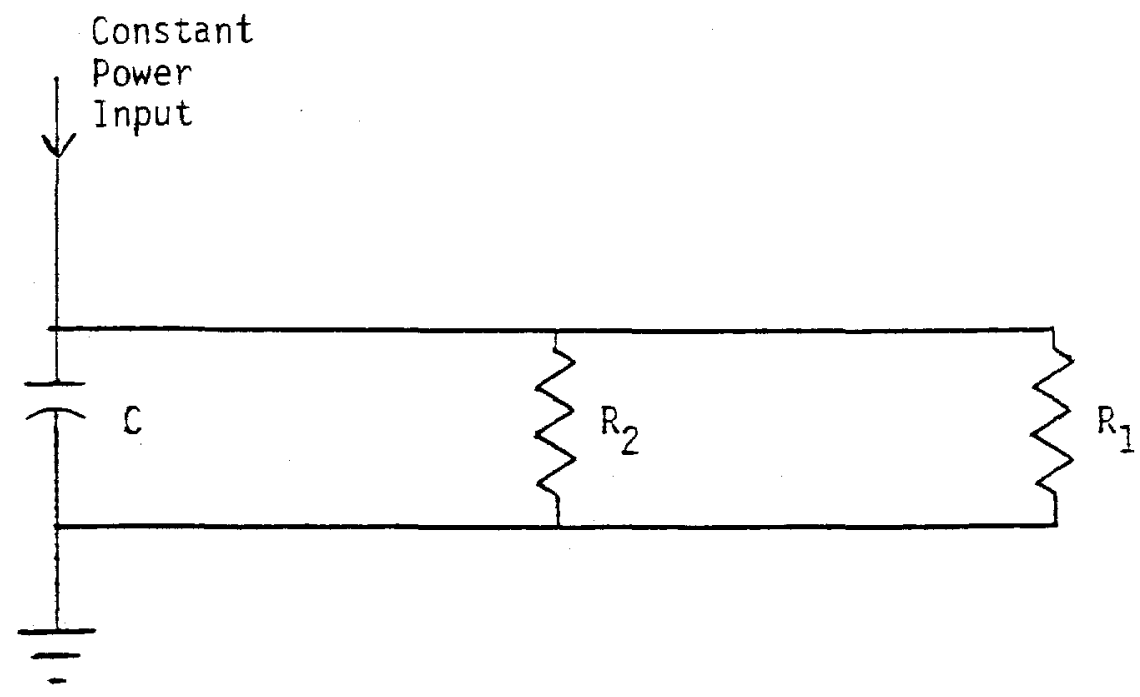

